MATHEMATICS OF COMPUTATION

Volume 73, Number 248, Pages 1801-1825

S 0025-5718(04)01660-6

Article electronically published on April 20, 2004

\title{
SPECTRAL-FRACTIONAL STEP RUNGE-KUTTA DISCRETIZATIONS FOR INITIAL BOUNDARY VALUE PROBLEMS WITH TIME DEPENDENT BOUNDARY CONDITIONS
}

\author{
I. ALONSO-MALLO, B. CANO, AND J. C. JORGE
}

\begin{abstract}
In this paper we develop a technique for avoiding the order reduction caused by nonconstant boundary conditions in the methods called splitting, alternating direction or, more generally, fractional step methods. Such methods can be viewed as the combination of a semidiscrete in time procedure with a special type of additive Runge-Kutta method, which is called the fractional step Runge-Kutta method, and a standard space discretization which can be of type finite differences, finite elements or spectral methods among others. Spectral methods have been chosen here to complete the analysis of convergence of a totally discrete scheme of this type of improved fractionary steps. The numerical experiences performed also show the increase of accuracy that this technique provides.
\end{abstract}

\section{INTRODUCTION}

Splitting, alternating directions or fractional steps [21, 16] 27] are well-known key words which refer to efficient discretization methods for complicated problems of mathematical physics in several variables. Such methods provide strong reductions of computational cost with respect to other classical, explicit or implicit methods. Beginning from the seminal works of Douglas, Peaceman and Rachford (see [14] 15. 24]), many authors extended and improved these methods, but most of them were developed and analyzed in close relation with specific classical evolutionary problems and using the concrete properties of their solutions. Soon it was seen that these kinds of methods suffer order reductions if they are used to integrate problems with time dependent boundary conditions (see [17); in order to avoid such reductions, the authors introduced corrections in some classical alternating direction schemes, which consist of modifying suitably the boundary conditions that the original methods suggest for their internal (fractionary) steps. The first idea which naturally arises for choosing boundary conditions in the internal steps comes from viewing the solutions of these steps as approaches in intermediate times

Received by the editor November 20, 2001 and, in revised form, December 30, 2002.

2000 Mathematics Subject Classification. Primary 65M20, 65M12; Secondary 65M60, 65J10.

Key words and phrases. Order reduction, fractional step Runge-Kutta methods, method of lines, partial differential equations, initial boundary value problems.

The first and second authors have obtained financial support from MCYT BFM 2001-2013 and JCYL VA025/01.

The third author has obtained financial support from the projects DGES PB97-1013, BFM2000-0803, a project of Gobierno de Navarra and a project of Universidad de La Rioja. 
between two time levels, but the evaluation of the boundary conditions at these intermediate times to define the internal steps is not necessarily the best option.

This order reduction drawback is also present in other classical discretization methods of initial boundary value problems with time dependent boundary conditions, for example, those derived from the combination of a Runge-Kutta method and a finite difference or finite element method to discretize the time and spatial variables, respectively. Such reductions have been deeply studied (see for example [4], [6], [22, [26]) and recently, Alonso-Mallo and Cano ([2], 3]) have developed and analyzed a technique which can be used in Runge-Kutta or Rosenbrock methods to avoid such order reduction. This technique also consists of modifying cleverly the boundary conditions naturally associated with the internal stages of these methods. This method has two great advantages: Firstly, the computational cost of modifying the boundary values is very cheap, compared with the cost of a complete stage. Secondly, only few changes are necessary in a standard software to accomplish these modifications (cf. [10], 20])

In this paper we prove that a similar technique can be implemented successfully to avoid these order reductions in the integration of initial boundary value problems with evolutionary boundary conditions with schemes of type alternating directions, splitting or fractional steps. The key for getting this in a unified way departs from the works of Jorge [18] and Jorge and Lisbona [19], where they prove that a method of these types may be viewed and analyzed as a combination of a standard spatial semidiscretization scheme and a time integration process which uses a special kind of additive Runge-Kutta method [13]. Such methods are called fractional step Runge-Kutta methods and we will refer to them as FSRK methods (see also Jorge and Bujanda [8]). These ideas have permitted not only the analysis of classical alternating direction, splitting or fractional step schemes in a unified way, but also their application to other problems as well as the development of new higher order fractional step schemes (see [9]).

For carrying out the development and analysis of our schemes, we will first consider, in the next section, an abstract initial boundary value problem formulated as an operational differential problem. Then we will study the convergence of a fractional step Runge-Kutta time semidiscretization, with improved boundary conditions for its internal stages. We prove in Sections 3 and 4 that such improvement permits that the method not have order reductions due to the influence of the boundary conditions.

The second discretization stage that we have performed to arrive at a numerical method consists of discretizing in space the stationary problems resulting from the application of an FSRK method to the continuous problem. Spectral methods have been chosen here due to their facilities for reaching high orders of convergence. Section 5 is devoted to the analysis of the fully discrete schemes derived of the use of a spectral method for the spatial discretization. More classical spatial discretization procedures can be seen combined with FSRK methods in [11] and [12.

Some numerical experiences are shown in Section 6 in order to illustrate the improvements provided by the technique introduced here of modifying the boundary conditions of the internal stages. Finally, some conclusions are made in Section 7. 


\section{NOTATION AND PRELIMINARIES}

Let $X$ and $Y$ be two Hilbert spaces. The scalar product in $X$ is denoted by $\langle\cdot, \cdot\rangle$. Let $A: D(A) \subset X \rightarrow X$ and $\partial: D(A) \subset X \rightarrow Y$ be two linear operators. We study in the present paper full discretizations of the abstract nonhomogeneous linear initial boundary value problem

$$
\begin{aligned}
u^{\prime}(t) & =A u(t)+f(t), & & 0 \leq t \leq T, \\
u(0) & =u_{0} \in X, & & \\
\partial u(t) & =g(t) \in Y, & & 0 \leq t \leq T .
\end{aligned}
$$

In practice, $X$ is a space of functions defined in a domain $\Omega \subset \mathbb{R}^{d}$ with regular boundary $\Gamma, d \geq 1, A$ is an unbounded differential operator defined in a subspace $D(A)$ formed by regular elements of $X$ and $\partial$ is a trace operator onto the space $Y$. The restriction of $A$ to $D\left(A_{0}\right)=$ Ker $\partial$, the elements of $D(A)$ with vanishing boundary, is denoted by $A_{0}$. (An example would be $A=\Delta, D(A)=H^{2}(\Omega)$, $D\left(A_{0}\right)=H^{2}(\Omega) \cap H_{0}^{1}(\Omega)$.) We make the following hypotheses:

(A1) The operator $-A_{0}$ is maximal and coercive, i.e.,

$$
\left\{\begin{array}{l}
\forall u \in X, \exists x \in D\left(A_{0}\right) \text { such that } x-A_{0} x=u, \text { and } \\
\forall x \in D\left(A_{0}\right),\left\langle-A_{0} x, x\right\rangle \geq 0 .
\end{array}\right.
$$

(A2) There exists an extension operator $K: Y \rightarrow D(A)$ such that $\partial K v=v$ for each $v \in Y$.

For instance, these hypotheses are satisfied when (2.1) is well posed in the sense defined in 23].

Since we are interested in approximations of high order, we make the following hypotheses of regularity of the solution of (2.1): There exists an integer number $r \geq 1$ such that

$$
A^{r-j} u^{(j)} \in C([0, T], X), \quad 0 \leq j \leq r .
$$

We remark that the assumption (2.2) implies that the time derivatives of the solution are regular in space, but without imposing any restriction on the boundary values. In fact, Theorem 3.1 in [1] shows that (2.2) is satisfied when the data $u_{0}$, $f$ and $g$ are regular and the boundary values $\partial u_{0}, \partial f(0)$ and $g(0)$ satisfy certain natural compatibility constraints. As a consequence, we obtain

$$
A^{j} u(t)=u^{(j)}(t)-\sum_{i=0}^{j-1} A^{j-i-1} f^{(i)}(t), \quad 0 \leq j \leq r,
$$

and by applying the boundary operator,

$$
\partial A^{j} u(t)=g^{(j)}(t)-\sum_{i=0}^{j-1} \partial A^{j-i-1} f^{(i)}(t), \quad 0 \leq j \leq r .
$$

These boundary values are used in this paper in order to define suitable boundary values of the internal stages of a fractional step Runge-Kutta (FSRK) method. Notice that the right-hand side of (2.4) is given only in terms of the data of (2.1).

Since we want to use FSRK methods for the time discretization of (2.1), we suppose that

$$
A=\sum_{i=1}^{m} A_{i}, \quad f=\sum_{i=1}^{m} f_{i}
$$


where $A_{i}: D\left(A_{i}\right) \subset X \rightarrow X$ are linear operators, simpler than $A$ from some points of view, such that $D(A)=\bigcap_{i=1}^{m} D\left(A_{i}\right)$. For example, following the classical alternating direction framework, $m$ will be the spatial dimension of the original evolution problem $(d)$ and operators $A_{i}$ will contain the partial derivatives with respect to one of the spatial variables $x_{1}, x_{2}, \ldots, x_{d}$. The techniques in this paper require that we also assume that

$$
A_{l_{1}}^{s_{l_{1}}} \cdots A_{l_{t}}^{s_{l_{t}}} u \in D\left(A_{i}\right)
$$

whenever $l_{1}, \ldots, l_{t}, i \in\{1, \ldots, m\}$, and $s_{l_{1}}+\cdots+s_{l_{t}} \leq r-1$. Moreover, we suppose that there exist linear operators $\partial_{i}: D\left(A_{i}\right) \subset X \rightarrow Y_{i}$ in such a way that Ker $\partial=$ $\bigcap_{i=1}^{m}$ Ker $\partial_{i}$ and the assumptions (A1) and (A2) also hold for each pair of operators $A_{i}, \partial_{i}, i=1, \ldots, m$; i.e., if $A_{0, i}$ is the restriction of $A_{i}$ to $D\left(A_{0, i}\right)=\operatorname{Ker} \partial_{i}$, the elements of $D\left(A_{i}\right)$ with vanishing boundary $\partial_{i}$, the following hypotheses hold:

(A1i) The operator $-A_{0, i}$ is maximal and coercive, i.e.

$$
\left\{\begin{array}{l}
\forall u \in X, \exists x \in D\left(A_{0, i}\right) \text { such that } x-A_{0, i} x=u, \text { and } \\
\forall x \in D\left(A_{0, i}\right),\left\langle-A_{0, i} x, x\right\rangle \geq 0 .
\end{array}\right.
$$

(A2i) There exists an extension operator $K_{i}: Y_{i} \rightarrow D\left(A_{i}\right)$ such that $\partial_{i} K_{i} v=v$ for each $v \in Y_{i}$.

For the time discretization of (2.1) we consider a special type of $s$-staged additive Runge-Kutta method with Butcher's table

\begin{tabular}{l|l|l|l|l}
$c$ & $\mathcal{A}_{1}$ & $\mathcal{A}_{2}$ & $\cdots$ & $\mathcal{A}_{m}$ \\
\hline & $b_{1}^{T}$ & $b_{2}^{T}$ & $\cdots$ & $b_{m}^{T}$
\end{tabular}

where $b_{i}=\left[b_{i, 1}, \ldots, b_{i, s}\right]^{T}, c=\left[c_{1}, \ldots, c_{s}\right]^{T}$, and

$$
\mathcal{A}_{i}=\left[\begin{array}{ccc}
a_{i, 11} & \cdots & a_{i, 1 s} \\
\vdots & & \vdots \\
a_{i, s 1} & \cdots & a_{i, s s}
\end{array}\right] .
$$

Let us denote $\mathbf{1}=[1, \ldots, 1]^{T} \in \mathbb{R}^{s}, c^{l}=\left[c_{1}^{l}, \ldots, c_{s}^{l}\right]^{T}$, for $l \geq 0$, and by $\mathcal{I}$ the $s$-dimensional identity matrix. From now on, we suppose that (2.7) has classical order $p$; we remember [18] that the order conditions

$$
b_{i_{1}}^{T} \mathcal{A}_{i_{2}} \cdots \mathcal{A}_{i_{j}} c^{l}=\frac{l !}{(j+l) !}, \quad\left\{\begin{array}{l}
1 \leq j \leq p, \\
0 \leq l \leq p-j, \\
i \bullet\{1, \ldots, m\},
\end{array}\right.
$$

are necessary and sufficient so that (2.7) has classical order $p$ when it is applied to a linear nonhomogeneous ordinary differential equation with time independent coefficients similar to (2.1).

We also use the stage order of (2.7) as the maximum integer positive number $q$ such that

$$
c^{l}=l \mathcal{A}_{i} c^{l-1}, l=1, \ldots, q, i=1, \ldots, m .
$$

This definition of stage order is similar to the one used for Runge-Kutta methods. We remark that this stage order is usually very small for known FSRK methods, usually 0 or 1 . As a consequence, an FSRK method may suffer a severe order reduction when the solution does not vanish at the boundary and the time stepsize is not too small. In this paper we use a similar technique to [2] and [3] to avoid this trouble. 
For $n>0$ integer, if we consider a constant time step $k>0$, we take $t_{n}=n k \leq T$. The application of an additive Runge-Kutta method, given by (2.7), to discretize in time (2.1), permits us to obtain approximations $u_{n}$ of the exact solution $u\left(t_{n}\right)$ by using the following process:

Firstly, for the internal stages, we obtain the equations

$$
\begin{aligned}
\left(\mathcal{I} \otimes I-\sum_{i=1}^{m}\left(\mathcal{A}_{i} \otimes k A_{i}\right)\right) U_{n} & =(\mathbf{1} \otimes I) u_{n}+k \sum_{i=1}^{m}\left(\mathcal{A}_{i} \otimes I\right) F_{i, n+c} \\
\left(\partial_{1}, \ldots, \partial_{m}\right) U_{n} & =\left(G_{1, n}, \ldots, G_{m, n}\right)
\end{aligned}
$$

where $U_{n}=\left[U_{n}^{1}, \ldots, U_{n}^{s}\right]^{T}$, the vector of internal stages,

$$
F_{i, n+c}=\left[f_{i}\left(t_{n}+c_{1} k\right), \ldots, f_{i}\left(t_{n}+c_{s} k\right)\right]^{T} \quad \text { and } \quad G_{i, n}=\left[\partial_{i} U_{n}^{1}, \ldots, \partial_{i} U_{n}^{s}\right]^{T}
$$

are the boundary values.

The time semidiscretization is achieved with the final equation of the additive Runge-Kutta method,

$$
u_{n+1}=u_{n}+\sum_{i=1}^{m}\left(b_{i}^{T} \otimes k A_{i}\right) U_{n}+k \sum_{i=1}^{m}\left(b_{i}^{T} \otimes I\right) F_{i, n+c} .
$$

If we want to obtain from an additive Runge-Kutta method the cost reduction typical of the fractional step schemes and preserve the unconditional convergence properties of classical implicit methods, the following restrictions on the coefficients of this method must be imposed:

Definition 2.1. A fractional step Runge-Kutta method (FSRK) is an additive RK method satisfying

$$
\left\{\begin{array}{l}
a_{l, i i} \geq 0, i \in\{1, \ldots, s\}, l \in\{1, \ldots, m\}, \quad a_{l, i j}=0, \forall j>i, \\
\left|b_{l, j}\right|+\sum_{i=1}^{s}\left|a_{l, i j}\right| \neq 0 \Rightarrow \sum_{\substack{\lambda=1 \\
\lambda \neq l}}^{m}\left(\left|b_{\lambda, j}\right|+\sum_{i=1}^{s}\left|a_{\lambda, i j}\right|\right)=0, \quad j \in\{1, \ldots, s\} .
\end{array}\right.
$$

Using these coefficient restrictions and the properties of the operators $A_{i}$ in [18, 8], it is proven that the calculus of (2.9a), (2.9b) has a unique solution. Besides, such a solution can be bounded independently of the size of $k$ for those FSRK methods which have all of their stages implicit (i.e., $\sum_{l=1}^{m} a_{l, i i}>0$ for $i=1, \ldots, s$ ) or those FSRK methods which have only the first stage explicit and a suitable last stage. These two cases contain most of the interesting classical and modern fractionary step schemes.

\section{Consistency of the time Semidiscretization}

Now, we study the behaviour of local errors of the semidiscrete method defined by (2.9a) and (2.9b). We denote by $\bar{U}_{n}=\left[\bar{U}_{n}^{1}, \ldots, \bar{U}_{n}^{s}\right]^{T}$ and $\bar{u}_{n+1}$ the values that satisfy (2.9a) and (2.9b) with $u_{n}=u\left(t_{n}\right)$. Then, the semidiscrete local truncation error in $t_{n}$ is defined by

$$
\rho_{n}=u\left(t_{n}\right)-\bar{u}_{n}, \quad 1 \leq n \leq N .
$$

Since $U_{n}$ are approximations of $U_{n+c}:=\left[u\left(t_{n}+c_{1} k\right), \ldots, u\left(t_{n}+c_{s} k\right)\right]^{T}$, a natural choice is $G_{i, n}=G_{i, n+c}:=\left[\partial_{i} u\left(t_{n}+c_{1} k\right), \ldots, \partial_{i} u\left(t_{n}+c_{s} k\right)\right]^{T}$. As we will see later, this choice is related to the usual full discretization of (2.1) by using the method of lines. However, with this choice, the order of the FSRK is only achieved when 
the solution satisfies certain artificial conditions at the boundary. Let us see how to avoid this order reduction phenomenon. We define

$$
\begin{aligned}
U_{n}^{[0]} & =U_{n+c}, \\
U_{n}^{[j+1]} & =(\mathbf{1} \otimes I) u\left(t_{n}\right)+\left(\sum_{i=1}^{m}\left(\mathcal{A}_{i} \otimes k A_{i}\right)\right) U_{n}^{[j]}+k \sum_{i=1}^{m}\left(\mathcal{A}_{i} \otimes I\right) F_{i, n+c},
\end{aligned}
$$

for each $j \geq 0$ and $1 \leq n \leq N$ and we consider the boundary values

$$
G_{i, n}^{[j]}=\partial_{i} U_{n}^{[j]},
$$

for $j \geq 0, i=1, \ldots, m$ and $1 \leq n \leq N$. When we apply the FSRK method given by (2.9a) and (2.9b) with $u_{n}=u\left(t_{n}\right)$ and (3.3) as boundary values of the internal stages, the corresponding values obtained in (2.9a) and (2.9b) are denoted by $\bar{U}_{n}^{[j]}$ and $\bar{u}_{n}^{[j]}$.

Theorem 3.1. Let $u$ be the solution of (2.1) satisfying (2.2) for $r=p+1$. We use the boundary values $G_{n}^{[j]}, 0 \leq j \leq p$, in (2.9a). Then the local errors $\rho_{n}^{[j]}=$ $u\left(t_{n}\right)-\bar{u}_{n}^{[j]}, 1 \leq n \leq N, 0 \leq j \leq p$, satisfy

$$
\left\|\rho_{n}^{[j]}\right\| \leq C k^{\bar{q}(j)+1}, \quad \text { for } k>0
$$

where $\bar{q}(j)=\min \{p, q+j\}$, and the constant $C$ depends only on the smoothness of $u$ and the FSRK method.

Proof. First, we take $j=0$ and we use $G_{i, n}=G_{i, n}^{[0]}$ to get

$$
\begin{aligned}
\left(\mathcal{I} \otimes I-\sum_{i=1}^{m}\left(\mathcal{A}_{i} \otimes k A_{i}\right)\right) \bar{U}_{n}^{[0]} & =(\mathbf{1} \otimes I) u\left(t_{n}\right)+k \sum_{i=1}^{m}\left(\mathcal{A}_{i} \otimes I\right) F_{i, n+c} \\
\left(\partial_{1}, \ldots, \partial_{m}\right) \bar{U}_{n}^{[0]} & =\left(G_{1, n}^{[0]}, \ldots, G_{m, n}^{[0]}\right) .
\end{aligned}
$$

Let $\delta_{n}^{[0]}$ be the value given by

$$
\left(\mathcal{I} \otimes I-\sum_{i=1}^{m}\left(\mathcal{A}_{i} \otimes k A_{i}\right)\right) U_{n}^{[0]}=(\mathbf{1} \otimes I) u\left(t_{n}\right)+k \sum_{i=1}^{m}\left(\mathcal{A}_{i} \otimes I\right) F_{i, n+c}+\delta_{n}^{[0]} .
$$

By expanding into Taylor series, $\delta_{n}^{[0]}$ is given by

$$
\begin{aligned}
\delta_{n}^{[0]}= & \sum_{j=q+1}^{p} \frac{k^{j}}{j !}\left[c^{j} u^{(j)}\left(t_{n}\right)\right. \\
& \left.\quad-j \sum_{i=1}^{m}\left(\left(\mathcal{A}_{i} c^{j-1} \otimes A_{i}\right) u^{(j-1)}\left(t_{n}\right)+\left(\mathcal{A}_{i} c^{j-1} \otimes I\right) f_{i}^{(j-1)}\left(t_{n}\right)\right)\right] \\
& +O\left(k^{p+1}\right) \\
= & O\left(k^{\bar{q}(0)+1}\right) .
\end{aligned}
$$

Denoting $\Delta_{n}^{[0]}=U_{n}^{[0]}-\bar{U}_{n}^{[0]}$ and subtracting (3.4) from (3.5.5),

$$
\begin{aligned}
\left(\mathcal{I} \otimes I-\sum_{i=1}^{m}\left(\mathcal{A}_{i} \otimes k A_{i}\right)\right) \Delta_{n}^{[0]} & =\delta_{n}^{[0]}, \\
\left(\partial_{1}, \ldots, \partial_{m}\right) \Delta_{n}^{[0]} & =0 .
\end{aligned}
$$


Therefore,

$$
\Delta_{n}^{[0]}=\left(\mathcal{I} \otimes I-\sum_{i=1}^{m}\left(\mathcal{A}_{i} \otimes k A_{i, 0}\right)\right)^{-1} \delta_{n}^{[0]}
$$

for $k>0$. On the other hand, since the FSRK method has order $p$,

$$
\begin{aligned}
& u\left(t_{n}\right)+\sum_{i=1}^{m}\left[\left(b_{i}^{T} \otimes k A_{i}\right) U_{n}^{[0]}+k\left(b_{i}^{T} \otimes I\right) F_{i, n+c}\right] \\
& =u\left(t_{n}\right)+\sum_{j=0}^{p-1} \frac{k^{j+1}}{j !} \sum_{i=1}^{m} b_{i}^{T} c^{j}\left(A_{i} u^{(j)}\left(t_{n}\right)+f_{i}^{(j)}\left(t_{n}\right)\right)+O\left(k^{p+1}\right) \\
& =u\left(t_{n}\right)+\sum_{j=0}^{p-1} \frac{k^{j+1}}{(j+1) !} \sum_{i=1}^{m}\left(A_{i} u^{(j)}\left(t_{n}\right)+f_{i}^{(j)}\left(t_{n}\right)\right)+O\left(k^{p+1}\right) \\
& =u\left(t_{n}\right)+\sum_{j=0}^{p-1} \frac{k^{j+1}}{(j+1) !} u^{(j+1)}\left(t_{n}\right)+O\left(k^{p+1}\right) \\
& =u\left(t_{n+1}\right)+O\left(k^{p+1}\right),
\end{aligned}
$$

where we have used the order conditions $b_{i}^{T} c^{j}=1 /(j+1), j=0, \ldots, p-1, i=$ $1, \ldots, m$.

Subtracting (2.9b), with $u_{n}=u\left(t_{n}\right)$ and $U_{n}=\bar{U}_{n}^{[0]}$ as internal stages, from (3.6), we get

$$
\rho_{n+1}^{[0]}=\sum_{i=1}^{m}\left(b_{i}^{T} \otimes k A_{i}\right)\left(\mathcal{I} \otimes I-\sum_{i=1}^{m} \mathcal{A}_{i} \otimes k A_{i, 0}\right)^{-1} \delta_{n}^{[0]}+O\left(k^{p+1}\right) .
$$

Using the coefficient restrictions (2.10) and the properties of the operators $A_{i}$, for more general initial boundary value problems, it is proven in [18 and 8] that the operator $\sum_{i=1}^{m}\left(b_{i}^{T} \otimes k A_{i}\right)\left(\mathcal{I} \otimes I-\sum_{i=1}^{m} \mathcal{A}_{i} \otimes k A_{i, 0}\right)^{-1}$ is bounded independently of the size of $k$, for two special classes of FSRK methods which includes most of the interesting classical and modern fractionary step schemes; from this, we obtain that $\rho_{n+1}^{[0]}=O\left(k^{\bar{q}(0)+1}\right)$.

Let us take $1 \leq j \leq p-q$. Then we have

$$
\begin{aligned}
\left(\mathcal{I} \otimes I-\sum_{i=1}^{m}\left(\mathcal{A}_{i} \otimes k A_{i}\right)\right) \bar{U}_{n}^{[j]} & =(\mathbf{1} \otimes I) u\left(t_{n}\right)+k \sum_{i=1}^{m}\left(\mathcal{A}_{i} \otimes I\right) F_{i, n+c}, \\
\left(\partial_{1}, \ldots, \partial_{m}\right) \bar{U}_{n}^{[j]} & =\left(G_{1, n}^{[j]}, \ldots, G_{m, n}^{[j]}\right)
\end{aligned}
$$

and

$$
\left(\mathcal{I} \otimes I-\sum_{i=1}^{m}\left(\mathcal{A}_{i} \otimes k A_{i}\right)\right) U_{n}^{[j]}=(\mathbf{1} \otimes I) u\left(t_{n}\right)+k \sum_{i=1}^{m}\left(\mathcal{A}_{i} \otimes I\right) F_{i, n+c}+\delta_{n}^{[j]}
$$


whence, by subtracting (3.2) from (3.8),

$$
\begin{aligned}
\delta_{n}^{[j]} & =U_{n}^{[j]}-U_{n}^{[j+1]} \\
& =\left(\sum_{i=1}^{m}\left(\mathcal{A}_{i} \otimes k A_{i}\right)\right)\left(U_{n}^{[j-1]}-U_{n}^{[j]}\right) \\
& =\left(\sum_{i=1}^{m}\left(\mathcal{A}_{i} \otimes k A_{i}\right)\right)^{j}\left(U_{n}^{[0]}-U_{n}^{[1]}\right) \\
& =\left(\sum_{i=1}^{m}\left(\mathcal{A}_{i} \otimes k A_{i}\right)\right)^{j} \delta_{n}^{[0]} \\
& =O\left(k^{q+j+1}\right) .
\end{aligned}
$$

We define

$$
u_{n+1}^{[j]}=u\left(t_{n}\right)+\sum_{i=1}^{m}\left(b_{i}^{T} \otimes k A_{i}\right) U_{n}^{[j]}+k \sum_{i=1}^{m}\left(b_{i}^{T} \otimes I\right) F_{i, n+c} .
$$

We deduce from (3.6) that $u_{n}^{[0]}=u\left(t_{n+1}\right)+O\left(k^{p+1}\right)$. Moreover, we have

$$
\begin{aligned}
u_{n+1}^{[j+1]}= & u\left(t_{n}\right)+\sum_{i=1}^{m}\left(b_{i}^{T} \otimes k A_{i}\right) U_{n}^{[j]}+k \sum_{i=1}^{m}\left(b_{i}^{T} \otimes I\right) F_{i, n+c} \\
& -\sum_{i=1}^{m}\left(b_{i}^{T} \otimes k A_{i}\right)\left(U_{n}^{[j]}-U_{n}^{[j+1]}\right) \\
= & u\left(t_{n}\right)+\sum_{i=1}^{m}\left(b_{i}^{T} \otimes k A_{i}\right) U_{n}^{[j]}+k \sum_{i=1}^{m}\left(b_{i}^{T} \otimes I\right) F_{i, n+c} \\
& -\sum_{i=1}^{m}\left(b_{i}^{T} \otimes k A_{i}\right) \delta_{n}^{[j]} \\
= & u_{n+1}^{[j]}-\sum_{i=1}^{m}\left(b_{i}^{T} \otimes k A_{i}\right) \delta_{n}^{[j]} .
\end{aligned}
$$

Now, we estimate the last term in the previous formula.

$$
\begin{aligned}
\sum_{i=1}^{m}\left(b_{i}^{T} \otimes k A_{i}\right) \delta_{n}^{[j]}= & \sum_{i=1}^{m}\left(b_{i}^{T} \otimes k A_{i}\right)\left(\sum_{l=1}^{m}\left(\mathcal{A}_{l} \otimes k A_{l}\right)\right)^{j} \delta_{n}^{[0]} \\
= & k^{j+1} \sum_{i, l_{1}, \ldots, l_{j}=1}^{m}\left(b_{i}^{T} \mathcal{A}_{l_{1}} \cdots \mathcal{A}_{l_{j}} \otimes A_{i} A_{l_{1}} \cdots A_{l_{j}}\right) \sum_{r=1}^{p} \frac{c^{r} k^{r}}{r !} u^{(r)}\left(t_{n}\right) \\
& -k^{j+1} \sum_{i, l_{1}, \ldots, l_{j}=1}^{m}\left(b_{i}^{T} \mathcal{A}_{l_{1}} \cdots \mathcal{A}_{l_{j}} \otimes A_{i} A_{l_{1}} \cdots A_{l_{j}}\right) \\
& \times r \sum_{r=1}^{p} \sum_{s=1}^{m}\left[\left(\mathcal{A}_{s} \otimes A_{s}\right) \frac{c^{r-1} k^{r}}{r !} u^{(r-1)}\left(t_{n}\right)\right. \\
& \left.+\left(\mathcal{A}_{s} \otimes I\right) \frac{c^{r-1} k^{r}}{r !} f_{s}^{(r-1)}\left(t_{n}\right)\right]+O\left(k^{p+j+1}\right)
\end{aligned}
$$




$$
\begin{aligned}
& \text { SPECTRAL-FRACTIONAL STEP RUNGE-KUTTA DisCRETIZATIONS } \\
& =\sum_{r=1}^{p} \frac{k^{j+1+r}}{r !}\left[\sum_{i, l_{1}, \ldots, l_{j}=1}^{m}\left(b_{i}^{T} \mathcal{A}_{l_{1}} \cdots \mathcal{A}_{l_{j}} c^{r} \otimes A_{i} A_{l_{1}} \cdots A_{l_{j}}\right) u^{(r)}\left(t_{n}\right)\right. \\
& -r \sum_{i, l_{1}, \ldots, l_{j}, s=1}^{m}\left(b_{i}^{T} \mathcal{A}_{l_{1}} \cdots \mathcal{A}_{l_{j}} \mathcal{A}_{s} c^{r-1} \otimes A_{i} A_{l_{1}} \cdots A_{l_{j}} A_{s}\right) u^{(r-1)}\left(t_{n}\right) \\
& \quad-r \sum_{i, l_{1}, \ldots, l_{j}, s=1}^{m}\left(b_{i}^{T} \mathcal{A}_{l_{1}} \cdots \mathcal{A}_{l_{j}} \mathcal{A}_{s} c^{r-1} \otimes A_{i} A_{l_{1}} \ldots A_{l_{j}}\right) f_{s}^{(r-1)}\left(t_{n}\right) \\
& \left.\quad+O\left(k^{p+j+1}\right)\right] \\
& =\sum_{r=q+1}^{p} \frac{k^{j+1+r}}{j+1+r !}\left[\sum_{i, l_{1}, \ldots, l_{j}=1}^{m}\left(\mathcal{I} \otimes A_{i} A_{l_{1}} \cdots A_{l_{j}}\right) u^{(r)}\left(t_{n}\right)\right. \\
& -\sum_{i, l_{1}, \ldots, l_{j}, s=1}^{m}\left(\mathcal{I} \otimes A_{i} A_{l_{1}} \cdots A_{l_{j}} A_{s}\right) u^{(r-1)}\left(t_{n}\right) \\
& \left.-\sum_{i, l_{1}, \ldots, l_{j}, s=1}^{m}\left(\mathcal{I} \otimes A_{i} A_{l_{1}} \cdots A_{l_{j}}\right) f_{s}^{(r-1)}\left(t_{n}\right)\right]+O\left(k^{p+j+1}\right) \\
& =\quad O\left(k^{q+j+1}\right),
\end{aligned}
$$

where we have used the order conditions (2.8).

Therefore, we obtain by using a recursive argument

$$
u_{n+1}^{[j]}=u\left(t_{n+1}\right)+O\left(k^{p+1}\right), \quad 0 \leq j \leq p-q .
$$

Now, we denote $\Delta_{n}^{[j]}=U_{n}^{[j]}-\bar{U}_{n}^{[j]}$. For $k>0$, we have

$$
\Delta_{n}^{[j]}=\left(\mathcal{I} \otimes I-\sum_{i=1}^{m}\left(\mathcal{A}_{i} \otimes k A_{i, 0}\right)\right)^{-1} \delta_{n}^{[j]} .
$$

We subtract (2.9b), with $u_{n}=u\left(t_{n}\right)$ and (3.3) as boundary values of the internal stages, from (3.11) written in the reversed order, and we get using (3.10)

$$
\begin{aligned}
\rho_{n+1}^{[j]} & =\sum_{i=1}^{m}\left(b_{i}^{T} \otimes k A_{i}\right)\left(\mathcal{I} \otimes I-\sum_{i=1}^{m} \mathcal{A}_{i} \otimes k A_{i, 0}\right)^{-1} \delta_{n}^{[j]}+O\left(k^{p+1}\right) \\
& =O\left(k^{\bar{q}(j)+1}\right) .
\end{aligned}
$$

In order to illustrate how this process works, we consider now a single example of the FSRK method which comes from the classical alternating direction method of Peaceman and Rachford [14, 15, 24] and which may be used for the time discretization of (2.1). This method is given by the array

$$
\begin{array}{c|ccc|ccc|}
0 & 0 & & & 0 & & \\
1 / 2 & 0 & 1 / 2 & & 1 / 2 & 0 & \\
1 & 0 & 1 & 0 & 1 / 2 & 0 & 1 / 2 \\
\hline & 0 & 1 & 0 & 1 / 2 & 0 & 1 / 2
\end{array}
$$


The corresponding system of equations for the internal stages $\left[U_{n}^{1}, U_{n}^{2}, U_{n}^{3}\right]^{T}$ may be written as

$$
\begin{aligned}
U_{n}^{1} & =u_{n}, \\
\left(1-\frac{k A_{1}}{2}\right) U_{n}^{2} & =u_{n}+\frac{k}{2}\left(A_{2} U_{n}^{1}+f_{2}\left(t_{n}\right)\right)+\frac{k}{2} f_{1}\left(t_{n+1 / 2}\right), \\
\left(1-\frac{k A_{2}}{2}\right) U_{n}^{3} & =u_{n}+k\left(A_{1} U_{n}^{2}+f_{1}\left(t_{n+1 / 2}\right)\right)+\frac{k}{2}\left(A_{2} U_{n}^{1}+f_{2}\left(t_{n}\right)\right)+\frac{k}{2} f_{2}\left(t_{n+1}\right),
\end{aligned}
$$

where, to obtain a unique solution, we need to assign the boundary values

$$
G_{1, n}^{2}=\partial_{1} U_{n}^{2}, \quad G_{2, n}^{3}=\partial_{2} U_{n}^{3}
$$

(Notice that, since the first internal stage $U_{n}^{1}$ is explicit, its boundary value is not necessary.)

The time discretization is achieved with the final equation

$$
u_{n+1}=u_{n}+k\left(A_{1} U_{n}^{2}+f_{1}\left(t_{n+1 / 2}\right)\right)+\frac{k}{2}\left(A_{2} U_{n}^{1}+f_{2}\left(t_{n}\right)\right)+\frac{k}{2}\left(A_{2} U_{n}^{3}+f_{2}\left(t_{n+1}\right)\right) .
$$

(Observe that, for this method, $u_{n+1}=U_{n}^{3}$.) This method has classical order $p=2$ and stage order $q=1$. In order to define the suitable boundary values of the internal stages, we consider

$$
\left[\begin{array}{c}
U_{n}^{1,[0]} \\
U_{n}^{2,[0]} \\
U_{n}^{3,[0]}
\end{array}\right]=\left[\begin{array}{c}
u\left(t_{n}\right) \\
u\left(t_{n+1 / 2}\right) \\
u\left(t_{n+1}\right)
\end{array}\right]
$$

and

$$
\left[\begin{array}{c}
U_{n}^{1,[1]} \\
U_{n}^{2,[1]} \\
U_{n}^{3,[1]}
\end{array}\right]=\left[\begin{array}{c}
u\left(t_{n}\right) \\
u\left(t_{n}\right)+\frac{k}{2}\left(A_{1} u\left(t_{n+1 / 2}\right)+f_{1}\left(t_{n+1 / 2}\right)\right)+\frac{k}{2}\left(A_{2} u\left(t_{n}\right)+f_{2}\left(t_{n}\right)\right) \\
u\left(t_{n}\right)+k\left(A_{1} u\left(t_{n+1 / 2}\right)+f_{1}\left(t_{n+1 / 2}\right)\right)+\frac{k}{2}\left(A_{2} u\left(t_{n}\right)+f_{2}\left(t_{n}\right)\right) \\
\quad+\frac{k}{2}\left(A_{2} u\left(t_{n+1}\right)+f_{2}\left(t_{n+1}\right)\right)
\end{array}\right]
$$

Our results prove order 1 when $G_{1, n}^{2}=\partial_{1} U_{n}^{2,[0]}=\partial_{1} u\left(t_{n+1 / 2}\right), G_{2, n}^{3}=\partial_{2} U_{n}^{3,[0]}=$ $\partial_{2} u\left(t_{n+1}\right)$ and order 2 when $G_{1, n}^{2}=\partial_{1} U_{n}^{2,[1]}, G_{2, n}^{3}=\partial_{2} U_{n}^{3,[1]}$. Notice that these boundaries make sense because of (2.6) . In fact, to obtain these boundary values in practice (without knowing the exact solution $u$ ), some trick must be used. It happens in many cases that, whenever $v \in D\left(A^{2}\right)$, it is possible to calculate $\partial_{1} A_{2} v$ (resp. $\partial_{2} A_{1} v$ ) as $C_{12} \partial_{1} v$ (resp. $C_{21} \partial_{2} v$ ), for some operators $C_{i j}: Y_{i} \rightarrow Y_{i}$. (Look at Section 5.1 in order to understand the hypotheses being made in that particular example.) In such a way, just $\partial_{1} u\left(t_{n}\right)$ is needed to calculate $\partial_{1} A_{2} u\left(t_{n}\right)$ for the second stage, and just $\partial_{2} u\left(t_{n+1 / 2}\right)$ is needed to calculate $\partial_{2} A_{1} u\left(t_{n+1 / 2}\right)$ for the third stage. For the rest of the terms needed, as it is usually not possible to calculate $\partial_{i} A_{i} v$ in the same direct way, the decomposition $A=A_{1}+A_{2}$ must be used, together with 
the fact that $A u=u^{\prime}-f$. Therefore,

$$
\left[\begin{array}{c}
U_{n}^{1,[1]} \\
U_{n}^{2,[1]} \\
U_{n}^{3,[1]}
\end{array}\right]=\left[\begin{array}{c}
u\left(t_{n}\right) \\
u\left(t_{n}\right)+\frac{k}{2}\left(u^{\prime}\left(t_{n+1 / 2}\right)-A_{2} u\left(t_{n+1 / 2}\right)-f_{2}\left(t_{n+\frac{1}{2}}\right)\right) \\
+\frac{k}{2}\left(A_{2} u\left(t_{n}\right)+f_{2}\left(t_{n}\right)\right) \\
u\left(t_{n}\right)+k\left(A_{1} u\left(t_{n+1 / 2}\right)+f_{1}\left(t_{n+1 / 2}\right)\right) \\
+\frac{k}{2}\left(u^{\prime}\left(t_{n}\right)-A_{1} u\left(t_{n}\right)-f_{1}\left(t_{n}\right)\right) \\
+\frac{k}{2}\left(u^{\prime}\left(t_{n+1}\right)-A_{1} u\left(t_{n+1}\right)-f_{1}\left(t_{n+1}\right)\right)
\end{array}\right],
$$

and from here the entire stage boundaries (3.14) can be calculated. For example, when the boundary data are $\partial_{1} u(t)=g_{1}(t)$ and $\partial_{2} u(t)=g_{2}(t)$,

$$
\begin{aligned}
\partial_{1} U_{n}^{2,[1]}= & G_{1, n}^{2,[1]} \\
= & g_{1}\left(t_{n}\right)+\frac{k}{2}\left(g_{1}^{\prime}\left(t_{n+1 / 2}\right)-C_{12} g_{1}\left(t_{n+1 / 2}\right)-\partial_{1} f_{2}\left(t_{n+\frac{1}{2}}\right)\right) \\
& +\frac{k}{2}\left(C_{12} g_{1}\left(t_{n}\right)+\partial_{1} f_{2}\left(t_{n}\right)\right), \\
\partial_{2} U_{n}^{3,[1]}= & G_{2,, n}^{3,[1]} \\
= & g_{2}\left(t_{n}\right)+k\left(C_{21} g_{2}\left(t_{n+1 / 2}\right)+\partial_{2} f_{1}\left(t_{n+1 / 2}\right)\right) \\
& +\frac{k}{2}\left(g_{2}^{\prime}\left(t_{n}\right)-C_{21} g_{2}\left(t_{n}\right)-\partial_{2} f_{1}\left(t_{n}\right)\right) \\
& +\frac{k}{2}\left(g_{2}^{\prime}\left(t_{n+1}\right)-C_{21} g_{2}\left(t_{n+1}\right)-\partial_{2} f_{1}\left(t_{n+1}\right)\right) .
\end{aligned}
$$

Remark 3.2. In other cases more iterations to the stage boundaries would be needed to achieve the optimal order. It is important to notice that (2.3), together with (2.5), the commutativity of the operators $\left\{A_{i}\right\}$, and the hypotheses that

$$
\partial_{i} A_{j}^{l} v=C_{i j}^{l} \partial_{i} v \text {, for every } v \in D\left(A^{l+1}\right) \text { and } i \neq j, 1 \leq l \leq r
$$

make that it is possible to calculate the stage boundaries from the given data $f$ and $g$. It is also remarkable that the hypotheses (3.15) can be assumed in most applications. Note also that, although operators $A_{i}$ do not commute, it will always be possible to calculate $G^{[1]}$ which ensures, at least, one order of improvement with respect to the one obtained with the classical boundary conditions, $G^{[0]}$.

\section{Stability And CONVERGence}

In order to obtain convergence to the solution of the continuous problem (2.1), for the semidiscrete solutions obtained with an FSRK method described by (2.9a), $(2.9 \mathrm{~b})$ with the coefficient restrictions (2.10), the consistency properties studied in the previous section must be accompanied by suitable stability properties. In this context, the classical concept of 0-stability is not applicable due to the fact that operators $A, A_{i}$ are generally not bounded. The natural stability requirement for our semidiscrete solutions is the preservation of the contractivity of the solutions of the continuous problem (2.1). This means that any two solutions $u(t)$ and $v(t)$ of the same problem (2.1), obtained by choosing two initial conditions $u_{0}$ and $v_{0}$, 
respectively, verify that $\left\|u\left(t^{\prime}\right)-v\left(t^{\prime}\right)\right\| \leq\|u(t)-v(t)\|, \forall t^{\prime} \geq t$. The preservation of this condition for our semidiscretization schemes means that

$$
\left\|u_{n+1}-v_{n+1}\right\| \leq\left\|u_{n}-v_{n}\right\|, \quad \forall n=0,1, \ldots
$$

and for any two sequences $\left\{u_{n}\right\}_{n}$ and $\left\{v_{n}\right\}_{n}$ generated with the same scheme (2.9a), (2.9b), from different initial values $u_{0}$ and $v_{0}$, respectively. If the set of operators $\left\{A_{0, i}\right\}_{i=0}^{m}$ is commutative, then the difference of numerical solutions $u_{n}-v_{n}$ satisfies the recurrence relation

$$
u_{n+1}-v_{n+1}=r\left(k A_{0,1}, k A_{0,2}, \ldots, k A_{0, m}\right)\left(u_{n}-v_{n}\right),
$$

where

$$
r\left(z_{1}, z_{2}, \ldots, z_{m}\right)=1+\sum_{i=1}^{m} z_{i} b_{i}^{T}\left(I-\sum_{j=1}^{m} z_{j} \mathcal{A}_{j}\right)^{-1} e
$$

is called the amplification function associated to the FSRK method. In this case, it is clear that the preservation of the contractivity for our FSRK schemes is equivalent to the transition operator is a contraction in $X$, i.e.,

$$
\left\|r\left(k A_{0,1}, k A_{0,2}, \ldots, k A_{0, m}\right)\right\| \leq 1 \text {. }
$$

In 19 the authors develop several results which ensure that this transition operator is a contraction if the rational function of $m$ complex variables $r\left(z_{1}, z_{2}, \ldots, z_{m}\right)$ is an $A$-acceptable rational approximation ${ }^{1}$ to the exponential $\exp \left(z_{1}+\cdots+z_{n}\right)$ joint to some additional assumptions on the commutative system $\left\{A_{0, i}\right\}_{i=0}^{m}$; moreover, if the operators $-A_{0, i}$ are coercive, some additional $A$-stability requirements on the FSRK methods of type strong $A$-stability, in particular the $L$-stability, ensures a strongest contractive behaviour of type $\left\|r\left(k A_{0,1}, k A_{0,2}, \ldots, k A_{0, m}\right)\right\| \leq \exp (-k \beta)$, where $\beta$ is a positive constant independent of the size of $k$. In [8], the authors face a more general evolutionary problem where the operators $A_{0, i}$ may change smoothly in time. In these cases a weaker stability result is obtained because the constant $\beta$ may have negative values, but this property is also sufficient to obtain convergence for the corresponding semidiscretization procedures, in finite intervals of time. For proving these stability results in such a quite general context, the holomorphic functional calculus in several variables is a strong tool. The penalty to pay is that the commutativity on the operators $A_{0, i}$ is a restriction difficult to overcome for theoretical proofs. Nevertheless, practice shows that this restriction is not necessary to get numerical stability. In [7] 9 the authors perform several examples considering noncommuting operators $A_{0, i}$ and numerical stability still remains.

The combination of the stability result (4.2) with the consistency result given in Theorem 3.1 permits us to obtain, via a well-known recurrence reasoning, the following convergence result.

Theorem 4.1. Let $u$ be the solution of (2.1) satisfying (2.2) for $r=p+1$, and let (2.9a), (2.9b) be an pth order FSRK method improved with the modified boundary conditions $G_{n}^{[j]}, 0 \leq j \leq p$. Then the global errors $R_{n}^{[j]}=u\left(t_{n}\right)-u_{n}^{[j]}$ satisfy

$$
\left\|R_{n}^{[j]}\right\| \leq C k^{\bar{q}(j)}, \quad \text { for } k>0,
$$

\footnotetext{
${ }^{1} A$-acceptability means that $\left|r\left(z_{1}, z_{2}, \ldots, z_{m}\right)\right| \leq 1$ for all $\left(z_{1}, z_{2}, \ldots, z_{m}\right) \in \mathbb{C}^{m}$ such that $\operatorname{Re}\left(z_{i}\right) \leq 0, \forall i=1, \ldots, m$. Weaker $A$-acceptability requirements of type $A(0)$-acceptability can be considered if the operators $A_{0, i}$ are self-adjoint.
} 
where $\bar{q}(j)=\min \{p, q+j\}$, and the constant $C$ depends only on the smoothness of $u$ and the coefficients of the FSRK method.

\section{Full DiscretizATion}

In this section we describe the space discretization of the elliptic problems which define each of the stages in (2.9a), and we analyze the error committed with the full discretization in space and time. We have considered spectral methods for the full discretization because they are very suitable for noncomplicated domains (as the ones in which we are interested in order to apply the advantages of fractional step Runge-Kutta methods) and because they are extremely accurate for regular solutions in such a way that, in the numerical experiments, the avoidance of order reduction in time is revealed even in the case of choosing few nodes for the spatial discretization.

5.1. Spatial discretization. Now we describe how the spectral method works to integrate an elliptic problem of the form

$$
\begin{aligned}
-A_{i} u & =f_{i}, \\
\partial_{i} u & =g_{i} .
\end{aligned}
$$

For simplicity, we will assume $i=1$, the domain is the square $\Omega=[-1,1] \times[-1,1]$ where $A_{1}=\partial_{x x}$ and $\partial_{1} u$ means the values of $u$ in

$$
\partial_{1} \Omega=\left\{\left(-1, y_{1}\right),\left(1, y_{2}\right) \text {, with } y_{1}, y_{2} \in[-1,1]\right\} .
$$

The approximated solution $u_{J}$ is searched among the polynomials of degree $\leq J$ $\left(\Pi_{J}\right)$ such that

$$
u_{J}=\tilde{R}_{1, J} u+I_{1, J} g_{1},
$$

where $\tilde{R}_{1, J} u, I_{1, J} g_{1} \in \Pi_{J}, \partial_{1}\left(\tilde{R}_{1, J} u\right)=0$ and $I_{1, J} g_{1}$ interpolates $g_{1}$ in $\Xi_{J} \cap \partial_{1} \Omega$ and vanishes in $\Xi_{J} \cap \stackrel{\circ}{\Omega}$, with $\Xi_{J}$ being the Gauss-Lobatto mesh with $J+1$ nodes in each direction.

Imitating the weak formulation of (5.1),

$$
\left\langle\nabla_{1} u, \nabla_{1} v\right\rangle=\left\langle f_{1}, v\right\rangle \text { for every } v \in H^{1}(\Omega) \text { such that } \partial_{1} v=0,
$$

the following is imposed:

$$
\left\langle\nabla_{1} u_{J}, \nabla_{1} v_{J}\right\rangle_{J}=\left\langle f_{1}, v_{J}\right\rangle_{J} \text { for every } v_{J} \in \Pi_{J} \text { such that } \partial_{1} v_{J}=0 \text {, }
$$

where $(\cdot, \cdot)_{J}$ is the Gauss-Lobatto quadrature rule in $\Omega$. That can be seen to be equivalent to the collocation method

$$
-A_{1} u_{J}=f \text { in }\left(\Omega \backslash \partial_{1} \Omega\right) \cap \Xi_{J} .
$$

Then, in 5 , it is proved that

$$
\left\|u\left(\cdot, y_{j}\right)-u_{J}\left(\cdot, y_{j}\right)\right\|_{L^{2}([-1,1])}=O\left(J^{2-m}\left\|u\left(\cdot, y_{j}\right)\right\|_{H^{m}([-1,1])}\right),
$$

where $\left\{y_{j}\right\}$ are the Gauss-Lobatto nodes. Therefore, applying Fubini's theorem, and the fact that the Gauss-Lobatto rule given by $\langle\cdot, \cdot\rangle_{J}$ approximates $\langle\cdot, \cdot\rangle_{L^{2}(\Omega)}$,

$$
\left\|u-u_{J}\right\|_{J}=O\left(J^{2-m}\|u\|_{H^{m}(\Omega)}\right) .
$$

Making afterwards the full discretization of the parabolic problem, the most important values are the interior ones. Therefore we shall denote as $R_{1, J}$ the polynomial which belongs to $X_{J}=\Pi_{J} \cap H_{0}^{1}(\Omega)$ and which interpolates $\tilde{R}_{1, J} u$ in 
the interior nodes of $\Xi_{J}$. Denoting also by $P_{J}: L^{2}(\Omega) \rightarrow X_{J}$ the projection operator with respect to $\langle\cdot, \cdot\rangle_{J}$ on $X_{J}$, it follows that

$$
\left\|P_{J} u-R_{1, J} u\right\|_{J} \leq\left\|u-u_{J}\right\|_{J}=O\left(J^{2-m}\|u\|_{H^{m}(\Omega)}\right),
$$

because $P_{J} u-R_{1, J} u$ vanishes in $\partial \Omega \cap \Xi_{J}$ and coincides with $P_{J} u-R_{1, J} u$ in $\stackrel{\circ}{\Omega} \cap \Xi_{J}$.

For the real implementation and analysis of (5.2), if we are just interested in the values of $u_{J}$ in $\stackrel{\circ}{\Omega} \cap \Xi_{J}$ (the same as those of $R_{1, J} u$ ), we can consider the collocation method in $(\Omega \backslash \partial \Omega) \cap \Xi_{J}$. That means that

$$
-P_{J} A_{1} \tilde{R}_{1, J} u-P_{J} A_{1} I_{1, J} g_{1}=P_{J} f .
$$

Now, as $\tilde{R}_{1, J} u=R_{1, J} u+I_{2, J} \partial_{2}\left(\tilde{R}_{1, J} u\right)$, and $P_{J} A_{1} I_{2, J} \partial_{2} \tilde{R}_{1, J} u=0$, (5.4) is saying that

$$
\text { - } A_{1, J, 0} R_{1, J} u-B_{1, J} g_{1}=P_{J} f
$$

where $A_{1, J, 0}: X_{J} \rightarrow X_{J}$ is defined by $A_{1, J, 0}=P_{J} A_{1}$ and $B_{1, J}: L^{2}\left(\partial_{1} \Omega\right) \rightarrow X_{J}$ by $B_{1, J}=P_{J} A_{1} I_{1, J}$. So (5.5) is enough to determine the $(J-1) \times(J-1)$ interior values in which we are interested.

5.1.1. Analysis of full discretization. As the values on $\partial \Omega$ are already known for the exact solution, the internal stages in (2.9a) must be discretized taking into account that we are just interested in the values of $u_{n}$ in the interior nodes of $\Xi_{J}$. From the previous section, if $U_{n, J, 0}, u_{n, J, 0} \in X_{J}$ are the approximations to $U_{n}$ in (2.9a) and $u_{n}$ in (2.9b) in the interior nodes, then

$$
\begin{array}{r}
\left(\mathcal{I} \otimes I_{J}-k \sum_{i=1}^{m}\left(\mathcal{A}_{i} \otimes A_{i, J, 0}\right)\right) U_{n, J, 0}=\left(\mathbf{1} \otimes I_{J}\right) u_{n, J, 0} \\
+k \sum_{i=1}^{m}\left(\mathcal{A}_{i} \otimes B_{i, J}\right) \partial_{i} U_{n}+k \sum_{i=1}^{m}\left(\mathcal{A}_{i} \otimes P_{J}\right) F_{i, n+c} \\
u_{n+1, J, 0}=u_{n, J, 0}+k \sum_{i=1}^{m}\left(b_{i}^{T} \otimes A_{i, J, 0}\right) U_{n, J, 0} \\
+k \sum_{i=1}^{m}\left(b_{i}^{T} \otimes B_{i, J}\right) \partial_{i} U_{n}+k \sum_{i=1}^{m}\left(b_{i}^{T} \otimes P_{J}\right) F_{i, n+c} .
\end{array}
$$

Remark 5.1. Because of the form of fractional step methods (2.10), whenever $B_{i, J} \partial_{i} U_{n, l}$ has been needed for one of the stages, no other $B_{j, J} \partial_{j} U_{n, l}$ is needed for $j \neq i$ in the following ones.

The following lemma will be used to assure that (5.6) is solvable and the operator $\left(\mathcal{I} \otimes I_{J}-k \sum_{i=1}^{m}\left(\mathcal{A}_{i} \otimes A_{i, J, 0}\right)\right)^{-1}$ is bounded. This statement is straightforward in case the method is $A$-stable and all the stages are implicit. The case in which the first stage is explicit is also interesting and must be studied with more care. That has already been done in [8].

Lemma 5.2. The operators $A_{i, J, 0}$ are dissipative.

Proof. For simplicity we will assume $i=1$ and $\Omega=[-1,1] \times[-1,1]$ and $A_{1}=\partial_{x x}$, although it can be generalized easily to other values of the index $i$, a bit more complicated domains and different operators $A_{i}$. 
If $u \in X_{J}$, then

$$
\left(A_{1, J, 0} u, u\right)_{J}=\left(P_{J} A_{1} u, u\right)_{J}=\left(A_{1} u, u\right)_{J} .
$$

On the other hand, if $\left\{L_{l}\right\}$ are the Lagrange polynomials associated to GaussLobatto nodes in $[-1,1]$, then for some constants $b_{l, k}$

$$
u=\sum_{l, k=1}^{J-1} b_{l, k} L_{l}(x) L_{k}(y)
$$

As a consequence, if $\left\{a_{j m}\right\}$ are the Gauss-Lobatto rule coefficients in $\Omega\left(a_{j m}=\right.$ $a_{j} a_{m},\left\{a_{j}\right\}$ Gauss-Lobatto coefficients in $[-1,1]$ ),

$$
\begin{aligned}
\left(A_{1} u, u\right)_{J} & =\sum_{j, m=1}^{J-1} a_{j m}\left[\sum_{l, k} b_{l, k} L_{l}^{\prime \prime}\left(x_{j}\right) L_{k}\left(y_{m}\right)\right]\left[\sum_{l, k} b_{l, k} L_{l}\left(x_{j}\right) L_{k}\left(y_{m}\right)\right] \\
& =\sum_{m=1}^{J-1} a_{m} \sum_{j=1}^{J-1} a_{j}\left[\sum_{l} b_{l, m} L_{l}^{\prime \prime}\left(x_{j}\right)\right]\left[\sum_{l} b_{l m} L_{l}\left(x_{j}\right)\right] \\
& =\sum_{m=1}^{J-1} a_{m} \int_{-1}^{1}\left(\sum_{l} b_{l, m} L_{l}^{\prime \prime}(x)\right)\left(\sum_{l} b_{l m} L_{l}(x)\right) d x \\
& =\sum_{m=1}^{J-1} a_{m}\left[\left.\left(\sum_{l} b_{l m} L_{l}^{\prime}(x)\right)\left(\sum_{l} b_{l m} L_{l}(x)\right)\right|_{-1} ^{1}-\int_{-1}^{1}\left(\sum_{l} b_{l m} L_{l}^{\prime}(x)\right)^{2} d x\right] \\
& =-\sum_{m=1}^{J-1} a_{m} \int_{-1}^{1}\left(\sum_{l} b_{l m} L_{l}^{\prime}(x)\right)^{2} d x .
\end{aligned}
$$

Here we have used that $\left[\sum_{l} b_{l, m} L_{l}^{\prime \prime}(x)\right]\left[\sum_{l} b_{l m} L_{l}(x)\right]$ is a polynomial of degree $\leq$ $2 J-2$ in the variable $x$ and the Gauss-Lobatto rule integrates it exactly. Finally, as the coefficients of Gauss-Lobatto are positive [25], (5.8) is saying that

$$
\left(A_{1} u, u\right)_{J} \leq 0 .
$$

Besides, $\left(A_{1} u, u\right)_{J}=0$ if and only if

$$
\sum_{l} b_{l m} L_{l}^{\prime}(x)=0, \quad m=1, \ldots, J-1 .
$$

As $L_{l}=0$ in $\{-1,1\}, \sum_{l} b_{l m} L_{l}(x)=0$ and therefore $b_{l m}$ and $u$ vanish.

Theorem 5.3. Because of (2.2), the exact solution $u$ of (2.1) belongs to $H^{m}(\Omega)$, with $m=2 r$. Then the full discretization given by (5.6) -(5.7) with $\partial_{i} U_{n}=G_{i, n}^{[j]}$ in (3.3) produces an estimate of the solution at the interior of $\Omega$ and at time $t_{n}$ $\left(u_{n, J, 0}\right)$ such that

$$
\left\|P_{J} u\left(t_{n}\right)-u_{n, J, 0}\right\|_{J}=O\left(n J^{2-m}+k^{\bar{q}(j)}+J^{4-m}\right) .
$$

Proof. For $g \in L^{2}(\partial \Omega)$, let $I_{J} g$ be the interpolant of $g$ at $\partial \Omega \cap \Xi_{J}$, which vanishes at $\stackrel{\circ}{\Omega} \cap \Xi_{J}$. Let us also define the set of polynomials of degree $\leq J$,

$$
\bar{U}_{n, J}=\bar{U}_{n, J, 0}+I_{J} \partial \bar{U}_{n, J}=\bar{U}_{n, J, 0}+I_{J} G_{n}^{[j]},
$$


as the solution of

$$
\begin{aligned}
(\mathcal{I} & \left.\otimes I_{J}-k \sum_{i=1}^{m}\left(\mathcal{A}_{i} \otimes A_{i, J, 0}\right)\right) \bar{U}_{n, J, 0} \\
& =\left(\mathbf{1} \otimes I_{J}\right) R_{J} u\left(t_{n}\right)+k \sum_{i=1}^{m}\left[\left(\mathcal{A}_{i} \otimes B_{i, J}\right) \partial_{i} U_{n, J}+\left(\mathcal{A}_{i} \otimes R_{J}\right) F_{i, n+c}\right]
\end{aligned}
$$

and $\bar{u}_{n+1, J, 0}$ in $X_{J}$ as

$$
\begin{aligned}
\bar{u}_{n+1, J, 0}= & R_{J} u\left(t_{n}\right)+k \sum_{i=1}^{m}\left(b_{i}^{T} \otimes A_{i, J, 0}\right) U_{n, J, 0} \\
& +k \sum_{i=1}^{m}\left(b_{i}^{T} \otimes B_{i, J}\right) \partial_{i} \bar{U}_{n, J}+k \sum_{i=1}^{m}\left(b_{i}^{T} \otimes R_{J}\right) F_{i, n+c} .
\end{aligned}
$$

On the other hand, from $\bar{U}_{n}^{[j]}$ in (3.7) (denoted as $\bar{U}_{n}$ for simplicity), $\bar{u}_{n}$ is defined as

$$
\bar{u}_{n+1}=u\left(t_{n}\right)+k \sum_{i=1}^{m}\left(b_{i}^{T} \otimes A_{i}\right) \overline{\mathbf{U}}_{n}+k \sum_{i=1}^{m}\left(b_{i}^{T} \otimes I\right) F_{i, n+c} .
$$

The proof is then based on the decomposition

$$
P_{J} u\left(t_{n+1}\right)-u_{n+1, J, 0}=\left(P_{J} u\left(t_{n+1}\right)-R_{J} u\left(t_{n+1}\right)\right)+\left(R_{J} u\left(t_{n+1}\right)-u_{n+1, J, 0}\right),
$$

where $R_{J} u$ is the elliptic projector operator which solves, according to the spectral discretization in use,

$$
\begin{aligned}
-A u & =f \text { in } \Omega, \\
\partial u & =g \text { in } \partial \Omega .
\end{aligned}
$$

That is to say, the operator $R_{J}: D(A) \rightarrow X_{J}$ is such that

$$
-A_{J, 0} R_{J} u-B_{J} g=P_{J} f
$$

where $A_{J, 0}=\left.P_{J} A\right|_{X_{J}}$ and $B_{J}=\left.P_{J} A I_{J}\right|_{L^{2}(\partial \Omega)}$. Because of classical results in [5],

$$
\left\|P_{J} u-R_{J} u\right\|_{J}=O\left(J^{2-m}\|u\|_{H^{m}(\Omega)}\right) .
$$

Therefore, it suffices to estimate the second brackets in (5.10), for which that is decomposed as

$$
\begin{aligned}
R_{J} u\left(t_{n+1}\right)-u_{n+1, J, 0}= & \left(R_{J} u\left(t_{n+1}\right)-R_{J} \bar{u}_{n+1}\right)+\left(R_{J} \bar{u}_{n+1}-\bar{u}_{n+1, J, 0}\right) \\
& +\left(\bar{u}_{n+1, J, 0}-u_{n+1, J, 0}\right) .
\end{aligned}
$$

Then, because of the definition of local truncation error $\rho_{n}$, (5.13) and Theorem 3.1 ,

$$
\begin{array}{r}
\left\|R_{J} u\left(t_{n+1}\right)-R_{J} \bar{u}_{n+1}\right\|_{J}=\left\|R_{J} \rho_{n}\right\|_{J}=\left\|\left(R_{J} \rho_{n}-P_{J} \rho_{n}\right)+P_{J} \rho_{n}\right\|_{J} \\
=O\left(J^{2-m}\left\|\rho_{n}\right\|_{H^{m}(\Omega)}+\left\|\rho_{n}\right\|_{J}\right)=O\left(J^{2-m}\|u\|_{H^{m}(\Omega)}+k_{n}^{\bar{q}(j)+1}\right) .
\end{array}
$$


To bound the second parentheses in (5.14), let us apply $R_{J}$ to (3.7) and we get that

$$
\begin{aligned}
R_{J} \bar{U}_{n}= & \left(\mathbf{1} \otimes R_{J}\right) u\left(t_{n}\right)+k \sum_{i=1}^{m}\left[\left(\mathcal{A}_{i} \otimes R_{J} A_{i}\right) \bar{U}_{n}+\left(\mathcal{A}_{i} \otimes R_{J}\right) F_{i, n+c}\right] \\
= & \left(\mathbf{1} \otimes R_{J}\right) u\left(t_{n}\right)+k \sum_{i=1}^{m}\left(\mathcal{A}_{i} \otimes A_{i, J, 0} R_{J}\right) \bar{U}_{n}+k \sum_{i=1}^{m}\left(\mathcal{A}_{i} \otimes R_{J}\right) F_{i, n+c} \\
& +k \sum_{i=1}^{m}\left(\mathcal{A}_{i} \otimes\left(R_{J} A_{i}-A_{i, J, 0} R_{J}\right)\right) \bar{U}_{n} \\
= & \left(\mathbf{1} \otimes R_{J}\right) u\left(t_{n}\right)+k \sum_{i=1}^{m}\left(\mathcal{A}_{i} \otimes A_{i, J, 0} R_{J}\right) \bar{U}_{n}+k \sum_{i=1}^{m}\left(\mathcal{A}_{i} \otimes R_{J}\right) F_{i, n+c} \\
& +k \sum_{i=1}^{m}\left(\mathcal{A}_{i} \otimes\left(\left(R_{J}-P_{J}\right) A_{i}+\left(P_{J} A_{i}-A_{i, J, 0} R_{J}\right)\right)\right) \bar{U}_{n} .
\end{aligned}
$$

Then,

$$
\begin{aligned}
& R_{J} \bar{U}_{n}-\overline{\mathbf{U}}_{n, J, 0}=\left[\mathcal{I} \otimes I_{J}-k \sum_{i=1}^{m}\left(\mathcal{A}_{i} \otimes A_{i, J, 0}\right)\right]^{-1} \\
& \times\left[k \sum_{i=1}^{m} \mathcal{A}_{i} \otimes\left(\left(R_{J}-P_{J}\right) A_{i}+\left(P_{J} A_{i}-A_{i, J, 0} R_{J}\right)\right) \bar{U}_{n}\right. \\
& \left.-k \sum_{i=1}^{m}\left(\mathcal{A}_{i} \otimes B_{i, J}\right) \partial_{i} \bar{U}_{n, J}\right] \\
& =\left[\mathcal{I} \otimes I_{J}-k \sum_{i=1}^{m}\left(\mathcal{A}_{i} \otimes A_{i, J, 0}\right)\right]^{-1} \\
& \quad \times\left[k \sum_{i=1}^{m} \mathcal{A}_{i} \otimes\left(-A_{i, J, 0} R_{J}+A_{i, J, 0} R_{i, J}+\left(R_{J}-P_{J}\right) A_{i}\right) \bar{U}_{n}\right] .
\end{aligned}
$$

On the other hand,

$$
\begin{aligned}
R_{J} \bar{u}_{n+1}= & R_{J} u\left(t_{n}\right)+k \sum_{i=1}^{m}\left(b_{i}^{T} \otimes R_{J} A_{i}\right) \bar{U}_{n}+k \sum_{i=1}^{m}\left(b_{i}^{T} \otimes R_{J}\right) F_{i, n+c} \\
= & R_{J} u\left(t_{n}\right)+k \sum_{i=1}^{m}\left(b_{i}^{T} \otimes A_{i, J, 0} R_{J}\right) \bar{U}_{n} \\
& +k \sum_{i=1}^{m}\left(b_{i}^{T} \otimes\left(R_{J} A_{i}-A_{i, J, 0} R_{J}\right)\right) \bar{U}_{n}+k \sum_{i=1}^{m}\left(b_{i}^{T} \otimes R_{J}\right) F_{i, n+c} \\
= & R_{J} u\left(t_{n}\right)+k \sum_{i=1}^{m}\left(b_{i}^{T} \otimes A_{i, J, 0} R_{J}\right) \bar{U}_{n} \\
& +k \sum_{i=1}^{m}\left(b_{i}^{T} \otimes\left(\left(R_{J}-P_{J}\right) A_{i}+\left(P_{J} A_{i}-A_{i, J, 0} R_{J}\right)\right)\right) \bar{U}_{n} \\
& +k \sum_{i=1}^{m}\left(b_{i}^{T} \otimes R_{J}\right) F_{i, n+c} .
\end{aligned}
$$


Therefore,

$$
\begin{aligned}
& R_{J} \bar{u}_{n+1}-\bar{u}_{n+1, J, 0} \\
& =\quad k\left(\sum_{i=1}^{m} b_{i}^{T} \otimes A_{i, J, 0}\right)\left(R_{J} \bar{U}_{n}-\bar{U}_{n, J, 0}\right) \\
& \quad+k \sum_{i=1}^{m}\left[b_{i}^{T} \otimes\left[\left(R_{J}-P_{J}\right) A_{i}+\left(P_{J} A_{i}-A_{i, J, 0} R_{J}\right)\right] \bar{U}_{n}-B_{i, J} \partial \bar{U}_{n, J}\right] \\
& =\quad k\left(\sum_{i=1}^{m} b_{i}^{T} \otimes A_{i, J, 0}\right)\left(R_{J} \bar{U}_{n}-\bar{U}_{n, J, 0}\right) \\
& \quad+k \sum_{i=1}^{m}\left[b_{i}^{T} \otimes\left(\left(R_{J}-P_{J}\right) A_{i}+A_{i, J, 0}\left(R_{i, J}-R_{J}\right)\right)\right] \bar{U}_{n} .
\end{aligned}
$$

Because of (5.13) and the definition of $\bar{U}_{n}$ in (3.7),

$$
\begin{aligned}
\left\|\left(R_{J}-P_{J}\right) A_{i} \bar{U}_{n}\right\|_{J} & =O\left(J^{2-(m-2)}\left\|A_{i} \bar{U}_{n}\right\|_{H^{m}(\Omega)}\right) \\
& =O\left(J^{4-m}\left\|\bar{U}_{n}\right\|_{H^{m}(\Omega)}\right)=O\left(J^{4-m}\|u\|_{H^{m}(\Omega)}\right) .
\end{aligned}
$$

It is also true that

$$
A_{i, J, 0}\left(R_{i, J}-R_{J}\right)=A_{i, J, 0}\left[\left(R_{i, J}-P_{J}\right)+\left(P_{J}-R_{J}\right)\right],
$$

and from here, attending to (5.3) and (5.13),

$$
\begin{aligned}
\left\|A_{i, J, 0}\left(R_{i, J}-R_{J}\right) \bar{U}_{n}\right\|_{J} & \leq\left\|A_{i, J, 0} O\left(J^{2-m}\left\|\bar{U}_{n}\right\|_{H^{m}(\Omega)}\right)\right\|_{J} \\
& =O\left(J^{4-m}\left\|\bar{U}_{n}\right\|_{H^{m}(\Omega)}\right) \\
& =O\left(J^{4-m}\|u\|_{H^{m}(\Omega)}\right) .
\end{aligned}
$$

Using now (5.15), (5.17), (5.18) and the uniform boundedness on $J$ and $k$ of $\left(\sum_{i=1}^{m} b_{i}^{T} \otimes k A_{i, J, 0}\right)\left(I \otimes I_{J}-k \sum_{i=1}^{m} \mathcal{A}_{i} A_{i, J, 0}\right)^{-1}$ [8], [18],

$$
R_{J} \bar{u}_{n+1}-\bar{u}_{n+1, J, 0}=O\left(k J^{4-m}\|u\|_{H^{m}(\Omega)}\right) .
$$

As for the third term of (5.14), subtracting (5.6) from (5.9), we get that

$$
\begin{aligned}
\bar{U}_{n, J, 0}-U_{n, J, 0}= & \left(\mathcal{I} \otimes I_{J}-k \sum_{i=1}^{m} \mathcal{A}_{i} \otimes A_{i, J, 0}\right)^{-1} \\
& \times\left[\left(\mathbf{1} \otimes I_{J}\right)\left(R_{J} u\left(t_{n}\right)-u_{n, J, 0}\right)+k \sum_{i=1}^{m}\left(\mathcal{A}_{i} \otimes\left(R_{J}-P_{J}\right)\right) F_{i, n+c}\right],
\end{aligned}
$$




$$
\begin{aligned}
\bar{u}_{n+1, J, 0}-u_{n+1, J, 0}= & R_{J} u\left(t_{n}\right)-u_{n, J, 0}+k \sum_{i=1}^{m}\left(b_{i}^{T} \otimes A_{i, J, 0}\right)\left(\bar{U}_{n, J, 0}-U_{n, J, 0}\right) \\
& +k \sum_{i=1}^{m}\left(b_{i}^{T} \otimes\left(R_{J}-P_{J}\right)\right) F_{i, n+c} \\
= & R_{J} u\left(t_{n}\right)-u_{n, J, 0} \\
& +k \sum_{i=1}^{m}\left(b_{i}^{T} \otimes A_{i, J, 0}\right)\left(\mathcal{I} \otimes I_{J}-k \sum_{i=1}^{m} \mathcal{A}_{i} \otimes A_{i, J, 0}\right)^{-1} \\
& \times\left[\left(\mathbf{1} \otimes I_{J}\right)\left(R_{J} u\left(t_{n}\right)-u_{n, J, 0}\right)\right. \\
& \left.+k \sum_{i=1}^{m}\left(\mathcal{A}_{i} \otimes\left(R_{J}-P_{J}\right)\right) F_{i, n+c}\right] \\
& +k \sum_{i=1}^{m}\left(b_{i}^{T} \otimes\left(R_{J}-P_{J}\right)\right) F_{i, n+c} .
\end{aligned}
$$

Now, (5.13) implies that

$$
\left(R_{J}-P_{J}\right) F_{i, n+c}=O\left(J^{2-(m-2)}\|f\|_{H^{m-2}(\Omega)}\right)=O\left(J^{4-m}\|u\|_{H^{m}(\Omega)}\right) .
$$

Using again that $\sum_{i=1}^{m}\left(b_{i}^{T} \otimes k A_{i, J, 0}\right)\left(\mathcal{I} \otimes I_{J}-k \sum_{i=1}^{m}\left(\mathcal{A}_{i} \otimes A_{i, J, 0}\right)\right)^{-1}$ is a bounded operator,

$$
\begin{aligned}
\bar{u}_{n+1, J, 0}-u_{n+1, J, 0}= & r\left(k A_{1, J, 0}, \ldots, k A_{m, J, 0}\right)\left(R_{J} u\left(t_{n}\right)-u_{n, J, 0}\right) \\
& +O\left(k J^{4-m}\|u\|_{H^{m}(\Omega)}\right),
\end{aligned}
$$

and as a consequence,

$$
R_{J} u\left(t_{n+1}\right)-u_{n+1, J, 0}=r\left(k A_{1, J, 0}, \ldots, k A_{m, J, 0}\right)\left(R_{J} u\left(t_{n}\right)-u_{n, J, 0}\right)+\epsilon_{J, n},
$$

where

$$
\epsilon_{J, n}=O\left(J^{2-m}+k^{\bar{q}(j)+1}+k J^{4-m}\right) .
$$

Using a recursion argument,

$$
\begin{aligned}
R_{J} u\left(t_{n}\right)-u_{n, J, 0}= & \sum_{l_{2}=0}^{n-1}\left(\prod_{l_{1}=l_{2}+1}^{n-1} r\left(k_{l_{1}} A_{1, J, 0}, \ldots, k_{l_{1}} A_{m, J, 0}\right) \epsilon_{J, l_{2}}\right) \\
& +\prod_{l_{1}=0}^{n-1} r\left(k_{l_{1}} A_{1, J, 0}, \ldots, k_{l_{1}} A_{m, J, 0}\right)\left(R_{J} u(0)-P_{J} u(0)\right) \\
= & O\left(n J^{2-m}+k^{\bar{q}(j)}+J^{4-m}\right),
\end{aligned}
$$

because of (4.2).

Remark 5.4. In practice, it happens many times that for moderate stepsizes $k_{n}$, the global error behaves in this way:

$$
P_{J} u\left(t_{n}\right)-u_{n, J, 0}=O\left(J^{2-m}+k^{\bar{q}(j)+1}+k J^{4-m}\right) .
$$

The reason for that was studied for Runge-Kutta methods in [22, 26] and it was mainly due to a summation-by-parts procedure which could be applied to the equivalent form of (5.20) for those methods. The same behaviour is observed for fractional step methods. 
Remark 5.5. The analysis carried out here for spectral methods can be extended easily to other spatial discretization methods. For example, if finite differences were considered, it would suffice to substitute in (5.5), (5.12) the operators $P_{J}, R_{J}$ by suitable restrictions to the corresponding nodes and $A_{J, 0}, A_{i, J, 0}, B_{i, J}$ by the corresponding matrices which result after the discretization of the elliptic boundary problems (5.11), (5.1). The error bounds obtained for (5.20) would depend on the particular methods considered.

\section{EXAMPLES AND NUMERICAL EXPERIMENTS}

To illustrate the results of the previous sections, we have performed some numerical experiments using the full discretization Spectral/FSRK described in (5.6)(5.7). We have considered the problem

$$
\begin{aligned}
u_{t}(t, x, y) & =u_{x x}(t, x, y)+u_{y y}(t, x, y)-4\left(1+y^{2}\right) e^{-t} e^{x+y^{2}} \text { in } \Omega=[-1,1] \times[-1,1], \\
u(0) & =e^{x+y^{2}}, \\
u(t, x, y) & =e^{-t} e^{x+y^{2}} \text { in } \partial \Omega,
\end{aligned}
$$

whose exact solution is $u(t, x, y)=e^{-t} e^{x+y^{2}}\left(\in H^{m}(\Omega)\right.$ for every integer $\left.m>0\right)$. In such a way, when considering $J=18$ nodes in each direction, the error coming from the space discretization in our numerical experiments is negligible in front of the error coming from the time semidiscretization, for moderate sizes of $k$, as was announced by the results of Theorem 5.3 .

As a first FSRK-method, we have considered the array (3.13). This method has stage order 1, according to (2.9) and classical order 2. Its amplification function is

$$
r\left(z_{1}, z_{2}\right)=\frac{1+\frac{z_{1}}{2}}{1-\frac{z_{1}}{2}} \frac{1+\frac{z_{2}}{2}}{1-\frac{z_{2}}{2}} .
$$

With the most natural boundary conditions for the stages $\left(G_{n}^{[0]}\right)$, the bounds in Theorem 3.1 and Remark 5.4 give order 2 for the local error and (a bit heuristically) for the global error. However, a better order can be observed with the boundary conditions for the stages $G_{n}^{[1]}$. In this case, the bound gives order 3 for local error and (a bit heuristically) for the global error. Table 1 shows the order for local error by integrating till time $T=k$ with timestep $k=1 / n$ and measuring the error at that particular time in $L^{2}$-norm approximated by the Gauss-Lobatto quadrature rule. Table2 2 does the same for global error integrating till time $T=1$ and measuring the

TABLE 1. Orders for local error and method (3.13)

\begin{tabular}{|c|c|c|c|c|c|c|}
\hline$n$ & $5-10$ & $10-20$ & $20-40$ & $40-80$ & $80-160$ & $160-320$ \\
\hline$G^{[0]}$ & 1.9569 & 2.0672 & 2.1377 & 2.1910 & 2.2400 & 2.2956 \\
\hline$G^{[1]}$ & 2.7992 & 2.8394 & 2.8715 & 2.8990 & 2.9223 & 2.9414 \\
\hline
\end{tabular}

TABLE 2. Orders for global error and method (3.13)

\begin{tabular}{|c|c|c|c|c|c|c|}
\hline$n$ & $5-10$ & $10-20$ & $20-40$ & $40-80$ & $80-160$ & $160-320$ \\
\hline$G^{[0]}$ & 2.1092 & 2.0035 & 1.9993 & 2.0007 & 2.0004 & 2.0001 \\
\hline$G^{[1]}$ & 2.3446 & 2.1490 & 2.0632 & 2.0287 & 2.0140 & 2.0070 \\
\hline
\end{tabular}




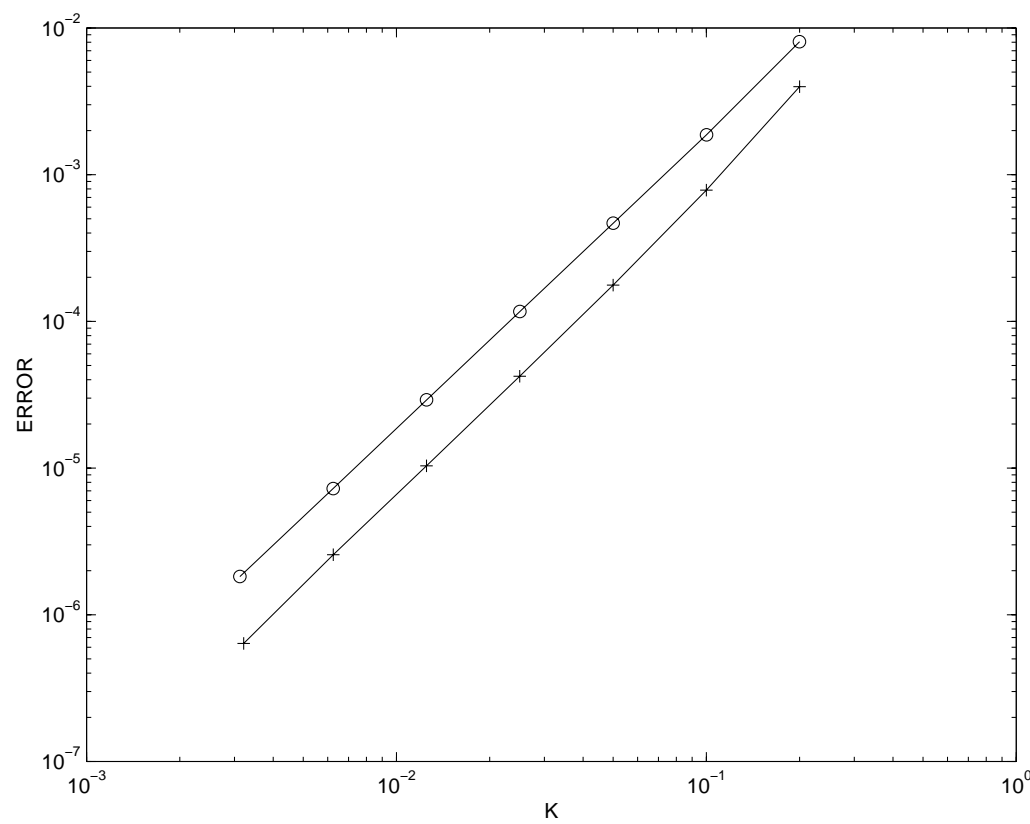

Figure 1. Comparison of efficiency with method (3.13) and stage boundaries $G_{n}^{[0]}$ and $G_{n}^{[1]}$.

error just at that final time. The orders observed are the expected ones. The only thing to notice is that the global order for $G_{n}^{[1]}$ is not as high as 3 for the smallest values of $k$. That corresponds to the fact that, for a fixed value of $J$ and $k$ small enough, the stiffness of the problem does not cause order reduction and the analysis can be done in the classical way. Consequently, no numerical order reduction will be observed in this case.

When looking at the size of the errors, the fact that the order is higher in general for $j=1$ leads to much smaller errors when $k$ is smaller. That can be observed in Figure 1 in which the $L^{2}$-norm is represented against the timestep.

TABLE 3. Orders for local error and method (6.1)

\begin{tabular}{|c|c|c|c|c|c|c|}
\hline$n$ & $5-10$ & $10-20$ & $20-40$ & $40-80$ & $80-160$ & $160-320$ \\
\hline$G^{[0]}$ & 0.7062 & 0.9920 & 1.1271 & 1.2103 & 1.3096 & 1.4811 \\
\hline$G^{[1]}$ & 2.4776 & 2.3838 & 2.2465 & 2.1781 & 2.1662 & 2.1579 \\
\hline$G^{[2]}$ & 3.1008 & 3.1365 & 3.1335 & 3.1492 & 3.1920 & 3.2619 \\
\hline
\end{tabular}

TABLE 4. Orders for global error and method (6.1)

\begin{tabular}{|c|c|c|c|c|c|c|}
\hline$n$ & $5-10$ & $10-20$ & $20-40$ & $40-80$ & $80-160$ & $160-320$ \\
\hline$G^{[0]}$ & 0.8470 & 1.0591 & 1.1561 & 1.2187 & 1.3010 & 1.4375 \\
\hline$G^{[1]}$ & 2.4887 & 2.3132 & 2.1861 & 2.1386 & 2.1370 & 2.1479 \\
\hline$G^{[2]}$ & 3.1374 & 3.1003 & 3.0636 & 3.0445 & 3.0363 & 3.0312 \\
\hline
\end{tabular}


Circles correspond to $G_{n}^{[0]}$ and crosses to $G_{n}^{[1]}$. As the last correction just means some modifications on the boundaries of the stages, the figure implies that that implementation is more competitive.

7]

As a second example, we have considered the FSRK-method of classical order 3

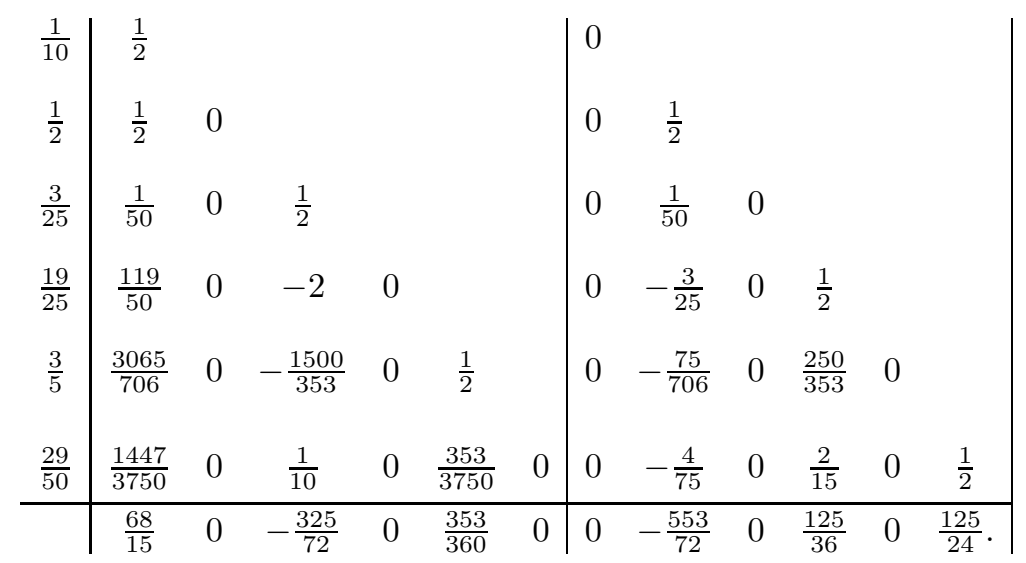

This method has stage order 0. Therefore, with $G_{n}^{[0]}$, Theorem 3.1 and Remark 5.4 give order 1 for local and global errors. That is observed in practice, as Tables 3] and 4 show. With $G_{n}^{[1]}$, theorems give order 2. In practice, the order is even a bit higher for the biggest values of the stepsize. With $G_{n}^{[2]}$, the expected order 3 for

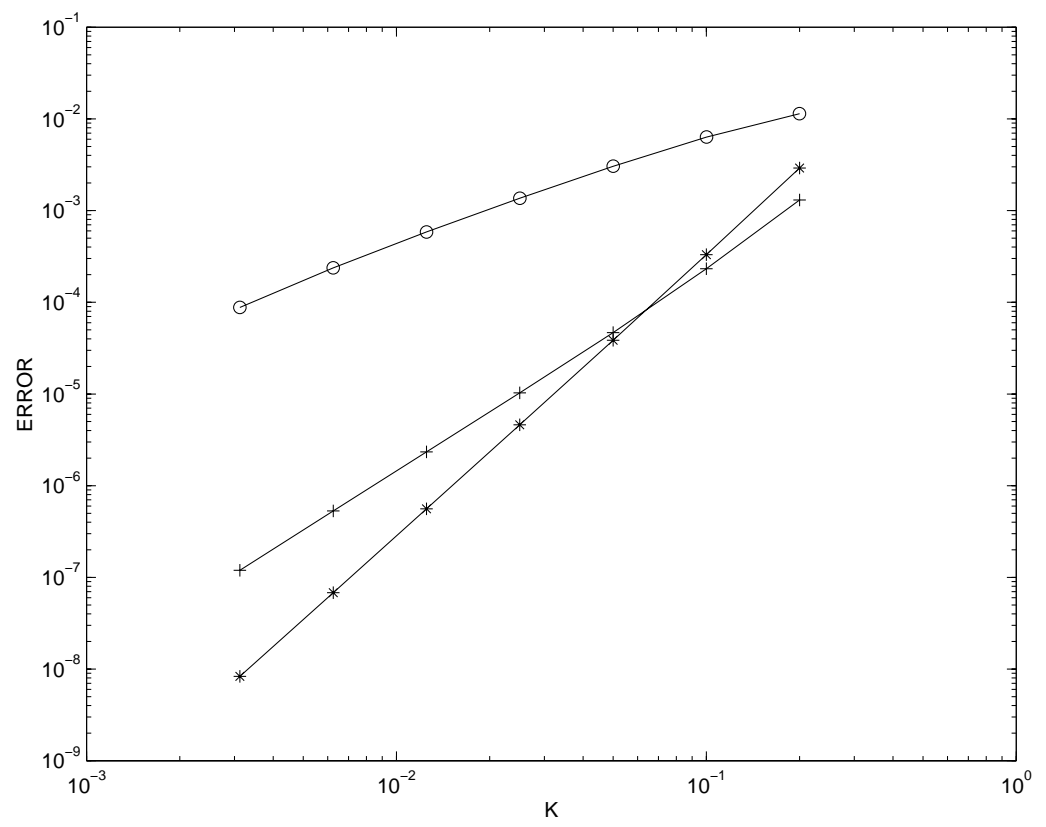

Figure 2. Comparison of efficiency with method (6.1) and stage boundaries $G_{n}^{[0]}, G_{n}^{[1]}$ and $G_{n}^{[2]}$. 


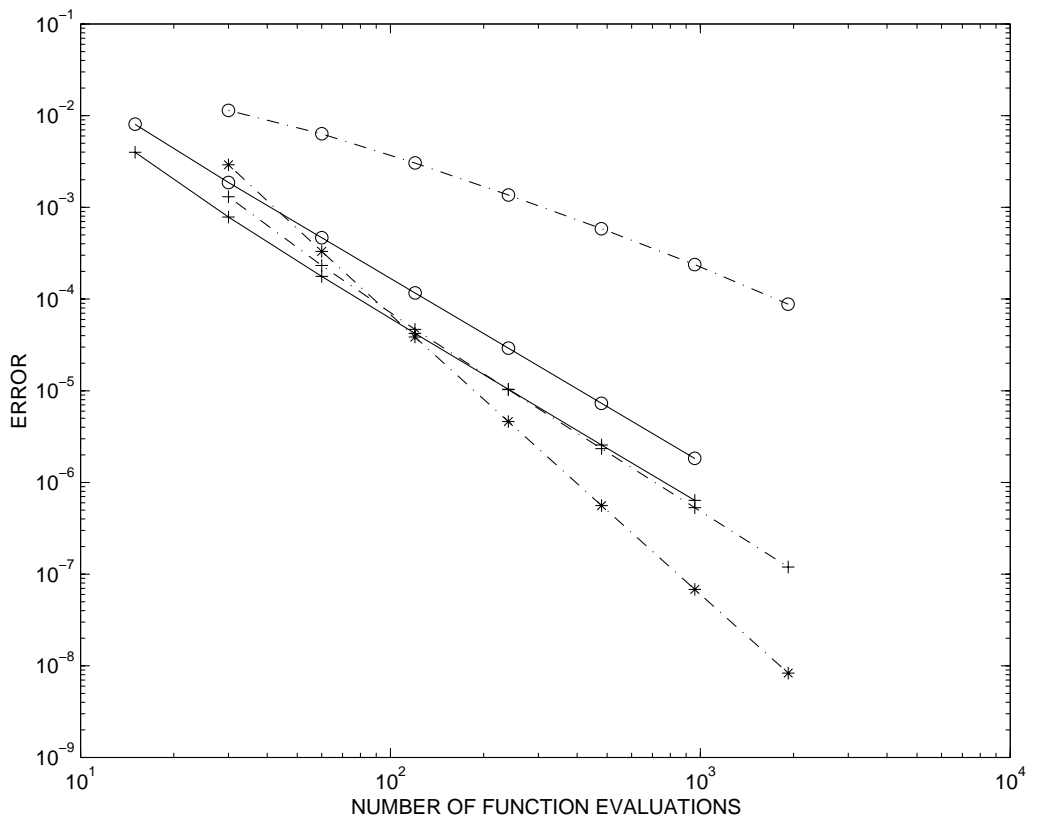

Figure 3. Number of evaluations the source term against the global errors with method (3.13) and stage boundaries $G_{n}^{[0]}$ and $G_{n}^{[1]}$, method (6.1) and stage boundaries $G_{n}^{[0]}, G_{n}^{[1]}$ and $G_{n}^{[2]}$.

both local and global error is observed in the tables. So, through the techniques of this paper, the classical order of the method has been reproduced for global error.

In Figure 2, we again represent global error against the timestep. The results for $G_{n}^{[0]}$ and $G_{n}^{[1]}$ are again represented by circles and crosses, respectively. Now, the results for $G_{n}^{[2]}$ are represented by asterisks. The gain in order with $j$ is again evident through the slopes of the lines in that figure. As for the size of the errors, the great decrease comes when changing $j=0$ for $j=1$. For all the values of $k$ which we have proved, the errors with one modification of the boundaries are several orders of magnitude smaller. Then, a bigger value of $j$ leads to smaller errors for the smallest values of the stepsize considered.

Finally, we have studied the computational cost of both methods with all the implementations considered. We remark that the performance of both methods improves clearly when the boundary values are successively modified. As expected, the method (3.13) (with $q=1$ ) is better than the method (6.1) (with $q=0$ ) when the usual implementation is used, corresponding to the use of $G_{n}^{[0]}$. When the stage boundaries are modified, (3.13) is still better than (6.1) for moderate values of the error (see Figure 3). However, (6.1) is better when a smaller value of the error is required.

\section{Conclusions}

We would like to mention now the important advantages of the type of discretization suggested here when integrating regular problems in noncomplicated domains. First of all, when dealing with the spectral discretization in space suggested here, 
fractional step Runge-Kutta methods manage to reduce the cost of the $L U$ factorization needed for the stages from $O\left(J^{6}\right)$ arithmetic operations in the Runge-Kutta case to $O\left(J^{3}\right)$ due to the fact that the FSRK time semidiscretization reduces the continuous problem to a set of essentially one-dimensional elliptic problems. Then, the forward and backward substitutions needed for the complete resolution of each stage means $O\left(J^{4}\right)$ operations in the Runge-Kutta case and just $O\left(J^{3}\right)$ in the fractionary step case. On the other hand, the spectral discretization in itself is very suitable for the same kind of problems, because it leads to really small errors in space. Finally, the fact that the reduction of order in time is completely avoided with the techniques in this paper leads to a very efficient method, mainly taking into account that the modifications needed to avoid that order reduction just mean $O(J)$ operations at each stage (the number of nodes in the boundary). This means nearly nothing compared with the total required to advance a step $\left(O\left(J^{3}\right)\right)$ and it is one of the features which makes this technique better than others already in the literature.

\section{REFERENCES}

[1] I. Alonso-Mallo, Rational methods with optimal order of convergence for partial differential equations, Appl. Numer. Math., 35 (2000), 265-292. MR 2001j:65129

[2] I. Alonso-Mallo, Runge-Kutta methods without order reduction for initial boundary value problems, Numer. Math. 91 (2002), 577-603. MR 2003e:65111

[3] I. Alonso-Mallo and B. Cano, Spectral/Rosenbrock discretizations without order reduction for linear parabolic problems, Appl. Numer. Math., 41 (2002), 247-268. MR 2003e:65163

[4] I. Alonso-Mallo and C. Palencia, Optimal orders of convergence in Runge-Kutta methods and linear initial boundary value problems, Appl. Numer. Math., 44 (2003), pp. 1-19. MR 2004a:65080

[5] C. Bernardy and Y. Maday, Approximations spectrales de problèmes aux limites elliptiques, Springer-Verlag France, Paris, 1992. MR 94f:65112

[6] P. Brenner, M. Crouzeix and V. Thomée, Single step methods for inhomogeneous linear differential equations in Banach spaces, Rairo Anal. Numer., 16 (1982), pp. 5-26. MR 83d:65268

[7] B. Bujanda, Métodos Runge-Kutta de Pasos Fraccionarios de orden alto para la resolución de problemas evolutivos de convección-difusión-reacción, Tesis, Universidad Pública de Navarra, 1999.

[8] B. Bujanda and J. C. Jorge, Stability results for fractional step discretizations of time dependent coefficient evolutionary problems, Appl. Numer. Math., 38 (2001), pp. 69-86. MR 2002c: 65150

[9] B. Bujanda and J. C. Jorge, Third order fractional step methods for multidimensional evolutionary convection-diffusion problems, Finite Difference Methods: Theory and Applications, Nova Science, (1999), 110-117. MR 2000e:65004

[10] M. P. Calvo and C. Palencia, Avoiding the order reduction of Runge-Kutta methods for linear initial boundary value problems, Math. Comput., 71 (2002), 1529-1543. MR 2003h:65091

[11] C. Clavero, J. C. Jorge, F. Lisbona and G. I. Shishkin, A fractional step method on a special mesh for the resolution of multidimensional evolutionary convection-diffusion problems, Appl. Numer., 27 (1998), 211-231. MR 99g:65089

[12] C. Clavero, J. C. Jorge, F. Lisbona and G. I. Shishkin, An alternating direction scheme on a nonuniform mesh for reaction-diffusion parabolic problems, IMA J. Numer. Anal., 20 (2000), 263-280. MR 2001b:65085

[13] G. J. Cooper and A. Sayfy, Additive Runge-Kutta methods for stiff ordinary differential equations, Math. Comput., 40 (1983), 207-218. MR 84b:65066

[14] J. Jr. Douglas, On the numerical integration of $\partial^{2} u / \partial x^{2}+\partial^{2} u / \partial y^{2}=\partial u / \partial t$ by implicit methods, J. Soc. Ind. Appl. Math. 3 (1955), 42-65. MR 17:196e

[15] J. Jr. Douglas and D. Peaceman, Numerical solution of two-dimensional heat flow problems, J. American Institute of Chemical Engineering Journal, 1 (1955), 505-512. 
[16] E. G. D'yaconov, Difference schemes with split operators for multidimensional unsteady problems, U.S.S.R. Comp. Math., 3 (1963), no. 4, 581-607.

[17] G. Fairweather and A. R. Mitchell, A new computational Procedure For A.D.I. Methods, SIAM Journal of Numer. Anal., Vol. 4, No. 2, (1967), 163-170. MR 36:1116

[18] J. C. Jorge, Los métodos de pasos fraccionarios para la integración de problemas parabólicos lineales: formulación general, análisis de la convergencia y diseño de nuevos métodos, Tesis, Universidad de Zaragoza, 1992.

[19] J. C. Jorge and F. Lisbona, Contractivity results for alternating direction schemes in Hilbert spaces, Appl. Numer. Math., 15 (1994), 65-75. MR 95f:65118

[20] S. Keeling, Galerkin/Runge-Kutta Discretizations for Parabolic Equations with TimeDependent Coefficients, Math. Comput., 52 (1989), 561-586. MR 90a:65239

[21] G. I. Marchuk, Splitting and Alternating Direction Methods. Handbook of Numerical Analysis vol. 1 (1990), Edited by P.G. Ciarlet and J.L. Lions, North Holland, 199-460. MR 91f:65001

[22] A. Ostermann and M. Roche, Runge-Kutta methods for partial differential equations and fractional orders of convergence, Math. Comput., 59 (1992), 403-420. MR 93a:65125

[23] C. Palencia and I. Alonso-Mallo, Abstract initial boundary values problems, Proc. Royal Soc. Edinburgh, 124A (1994), 879-908. MR 95k:34090

[24] D. W. Peaceman and H. H. Rachford, The numerical solution of parabolic and elliptic differential equations, J. SIAM., 3 (1955), 28-42. MR 17:196d

[25] A. Ralston, A first course in Numerical Analysis, McGraw-Hill, New York, 1965. MR 32:8479

[26] J. M. Sanz-Serna, J. G. Verwer, and W. H. Hundsdorfer, Convergence and Order Reduction of Runge-Kutta Schemes Applied to Evolutionary Problems in Partial Differential Equations, Numer. Math., 50 (1986), 405-418. MR 88f:65146

[27] N. N. Yanenko, The method of fractional steps, Springer, 1971. MR 46:6613

Departamento de Matemática Aplicada y Computación, Facultad de Ciencias, UniVERSidAd de VAlladolid, 47011 Valladolid, Spain

E-mail address: isaias@mac.uva.es

Departamento de Matemática Aplicada y Computación, Facultad de Ciencias, UniVERsidad de VAlladolid, 47011 Valladolid, Spain

E-mail address: bego@mac.uva.es

Departamento de Matemática e Informática, Universidad Pública de Navarra, 31006 PAMPlONA, SPAin

E-mail address: jcjorge@unavarra.es 